\title{
One and Two Electron Integrals over \\ Cartesian Gaussian Functions
}

Larry E. McMurchie

and

Ernest R. Davidson

Depantment of Chemistry BG-10

University of Washington

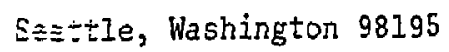

$$
0.0 .3549003
$$

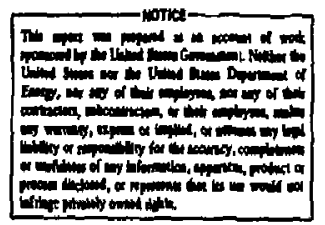


Abstract

A formalism is developed which allows overlap, kinetic energy, potentia? energy and electron repulsion integrals over cartesian Gaussian functions to be expressed in a very compact form involving easily computed auxiliary functions. Similar formulas involving the same auxiliary functions are given for the common charge moments, electric-field operators, and spin-interaction operators. Recursion relations are given for the auxiliary functions whic, make possible the use of Gaussian functions of arbitrarily large angular momentum. An algorithm is described for the computation of electron repulsion * inteミミミ?ร. 


\section{i. INTRODUCTION}

Cartesian Gaussian functions of the form $x_{A}^{n} y_{A}^{\ell} z_{A}^{m} \exp \left(-\alpha_{A} r_{A}^{2}\right)$ were first proposed as basis functions by Boys. ' The obvious exploitable advantage of Gaussian functions over Slater type orbitals (STO's) is the ease with which a product of Gaussizns on two different centers can be written as a simple function on a common center. ${ }^{2}$ In the 1960 's when calculations on diatomic systems were already common, the intractability of the four-center integral over STO's appeared to present a major block to polyatomic calculations. ${ }^{3}$ Gaussians began to enjoy increased popularity when it was found thet a fixed linear combination (a so-called "contracted Gaussias"; could be :sミd to approximate an atomic orbital to good accuracy. Intialiy $;$ : trend was to use combinations of simple Gaussi=n "icbes" $(::=:==0) .4$ The resulting electron repulision integrez tad been $3: \ldots . .-$ by Boys ${ }^{1,2,3}$ to involve only a square root, an expcential, and $-\ldots$ error function. Functions of $p$ or a type were azzoximated is

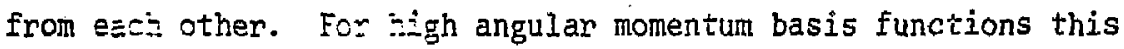
approaci jecomes intractable both because of the large numbers of lobes involved and because of large differencing errors in combining the integrals over the basic lobes.

The obvious alternative to. contracted gaussian lobes was contracted cartesian Gaussians. Basis sets involving these functions are now more-or-less standardized. ${ }^{5}$ Formulas for integrals over p-type Gaussians were easily derived ${ }^{2}$ and programined. Standard program packages such as POLYATOM ${ }^{6}$ and GAUSSIAN $70^{6}$ have been available for some time. Some versions of these packages have included $d$ and 
f orbitals. The formulas available for these integrals, ${ }^{2}$ while completely general, do not allow systematic calculation oi integrals for higher angular momentum. In this paper we present auxiliary functions and recursion relations from which integrals for all values of $n+\ell+m$ can be systematically (and efficiently) computed 


\section{CHARGE DISTRIBUTIONS}

An unnormalized Gaussian function on center A will be given by

$$
\phi\left(n, \ell, m, \alpha_{A}, A\right)=x_{A}^{n} y_{A}^{\ell} z_{A}^{m} \exp \left(-\alpha_{A} r_{A}^{2}\right)
$$

where $\left(x_{A}, y_{A}, z_{A}\right)$. are the components of the vector $\underline{r}_{A}=\underline{r}-\underline{A}$ with norm $r_{A}$ : The normalization constant for this function is

$$
N_{n \ell m}\left(\alpha_{A}\right)=N_{n}\left(\alpha_{A}\right) N_{l}\left(\alpha_{A}\right) N_{m}\left(\alpha_{A}\right)
$$

where

$$
N_{k}(\Omega)=(2 \alpha / \pi)^{? / 4}(4 \alpha)^{k / 2}[(2 k-1) ! !]^{-1 / 2}
$$

Such fusiotions are z=Esred to as $s, p, d, \ldots$ when $L=\Omega+\ell+\pi$ is $0, I, 2, \ldots, r \in \Sigma z=-i$ ively, Contracted basis functions can be forme $=0 \mathrm{~m}$ the $\phi^{\prime \prime}=$ rarious ways. For example, it is usual to defirs

$$
\sum_{i, 2 I A}=\sum_{\alpha_{A}} c^{i}\left(a_{n l m}\left(\alpha_{A}\right) \phi\left(n, l, m, \alpha_{A}, A\right)\right.
$$

where $C^{-}\left(a_{A}\right)$ is indegerdent of $n, l, m$ (for fixed $L$ ). Alternatively a more ganeral

$$
g_{L M A}=\sum_{\alpha_{A}} c^{L}\left(c_{A}\right) \sum_{n \ell m} B_{n \ell m}^{L M} \phi\left(n, \ell, \pi, \alpha_{A}, A\right)
$$

can be considered wich allows true angular momentum eigenfunctions to be formed (with the $\alpha$ dependent part of $\mathrm{N}_{n \ell m}(\alpha), \alpha^{(2 L+3) / 4}$, absorbed in $c^{L}\left(a_{A}\right)$ and the $\alpha$ independent factors absorbed into $\mathrm{B}_{n \ell m}^{\mathrm{LM}} \mathrm{H}$.

Efficient computation of integrals requires that $f_{n \ell m A}$ or $g_{\text {LMA }}$ which involve the same $\alpha^{\prime}$ s and same nucleus be treated as sets. 
For inaximum efficiency these sets should be large enough that calculation of the auxiliary functions common to a block of integrals becomes insignificant. On the other hand, excessively large blocks of integrals which resuit from treating all fifteen $L=4$ functions as one set should be avoided.

For the sake of simplicity, the formulas in this paper will be given only for integrals over $\phi^{\prime} s$ rather than $f^{\prime} s$ or $g^{\prime} s$, in order to avoid writing explicitly the sums over $\alpha$. To this end it is convenient to define the charge distribution $\Omega_{\text {IJ }}$ of two functions $\phi_{I}\left(n, l, m, \alpha_{A}, A\right)$ and $\phi_{J}\left(\bar{n}, \bar{l}, \overline{\bar{m}}, \alpha_{B}, B\right)$ as

$$
\Omega_{I J}=s_{I} \phi_{J}=x_{A}^{n} x_{B} \bar{n}_{A}^{\ell} y_{B}^{\bar{l}_{A}} z_{A}^{m_{B}} \bar{m} \exp \left[-\left(\alpha_{A} r_{A}^{2}+\alpha_{B} r_{B}^{2}\right)\right] \text {. }
$$

The rey to the vinity of Gausians is the well-known identing winch transzzos the above exponential to a single

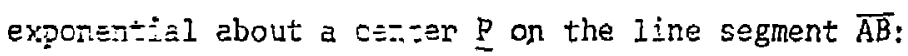

$$
\operatorname{exE}\left[-\left(\alpha_{A} r_{A}^{2}+\alpha_{B}{ }^{2} \equiv\right)\right]=E_{I J} \exp \left(-\alpha_{P} r_{P}^{2}\right)
$$

where

$$
\begin{aligned}
& \underline{P}=\left(\alpha_{A} \underline{A}+\alpha_{B} B\right) /\left(a_{A}+\alpha_{B}\right), \\
& \alpha_{P}=a_{A}+\alpha_{B},
\end{aligned}
$$

and

$$
E_{I J}=\exp \left\{-\alpha_{A} \alpha_{B}\left(\alpha_{A}+\alpha_{B}\right)^{-1}|\underline{A}-\underline{B}|^{2}\right\}
$$

Since molecular calculations usually require absolute rather than relative accuracy, all integrals involving $\Omega_{I J}$ can be neglected if the constant. $E_{I J}$ is sufficiently small.

The products $x_{A}^{n} x_{B}^{\bar{n}}, y_{A}^{l} y_{B}^{\bar{l}}$, and $z_{A}^{m_{B}} z_{B}^{m}$ could be converted into polynomials in $x_{p}, y_{p}$ and $z_{p}$ using relations like 


$$
x_{A}=x_{P}+\overline{P A}_{x}
$$

where

$$
\begin{aligned}
& x_{A}=x-A_{x}, \\
& x_{P}=x-P_{x},
\end{aligned}
$$

and

$$
\overline{P A}_{x}=P_{x}-A_{x}
$$

It is more convenient in what follows, however, to define

$$
\Lambda_{j}\left(x_{p} ; \alpha_{p}\right) \exp \left(-\alpha_{p} x_{p}^{2}\right)=\left(\frac{\partial}{\partial \tilde{P}_{x}}\right)^{j} \exp \left(-\alpha_{p} x_{p}^{2}\right),
$$

from which it follows that $\Lambda_{j}$ is related to the Hermite polynomial $\mathrm{H}_{\mathbf{j}}$ by

$$
\Lambda_{j}\left(x_{z} ; \alpha_{p}\right)=\alpha_{p}^{q / 2} \bar{z}_{j}\left(\alpha_{p}^{1 / 2} x_{p}\right) .
$$

The wility of the $A^{\prime} \equiv$ is obvious - they will allow the charge distrisution to be written as a sum of derivatives with respect to the coordinates of ? and these derivatives can be taken outside of any integral over electronic coordinates.

Now let us find the coefficients for expanding $x_{A}^{n} x_{B}^{n}$ in the $\Lambda$ 's:

$$
x_{A}^{n} x_{B}^{\bar{n}}=\sum_{N=0}^{n+\bar{n}} d_{N}^{n n_{n}^{n}} \Lambda_{N}\left(x_{p} ; \alpha_{P}\right) .
$$

The recursion relation for the Hermite polynomials is

$$
\xi \mathrm{H}_{N}(\xi)=\mathrm{NH}_{\mathrm{N}-1}(\xi)+\frac{\mathrm{T}}{2} \mathrm{H}_{\mathrm{N}+1}(\xi) \text {. }
$$

Consequentiy.

$$
x_{A} \Lambda_{N}\left(x_{P} ; \alpha_{P}\right)=N \Lambda_{N-1}+\overline{P A}_{x} \Lambda_{N}+\frac{1}{2} \Lambda_{N+1} / \alpha_{P}
$$


The recursion relations on the $d_{N}^{n \bar{n}}$ are then easily seen to be

$$
\begin{aligned}
& d_{N}^{n+1, \bar{n}}=\left(2 \alpha_{P}\right)^{-1} d_{N-1}^{n \bar{n}}+\overline{P A}_{x} d_{N}^{n \bar{n}}+(N+1) d_{N+1}^{n \bar{n}}, \\
& d_{N}^{n, \bar{n}+1}=\left(2 \alpha_{P}\right)^{-1} d_{N-1}^{n \bar{n}}+\overline{P B}_{x} d_{N}^{n \bar{n}}+(N+1) d_{N+1}^{n \bar{n}},
\end{aligned}
$$

where

$$
\oint^{0}=1 \text {. }
$$

Similarly we can write

and

$$
y_{A}^{\ell} y_{B}^{\bar{\ell}}=\sum_{L=0}^{\ell+\bar{l}} e_{L}^{l \bar{l}} \Lambda_{L}\left(y_{P} ; \alpha_{P}\right)
$$

$$
z_{A}^{m} z_{B}^{\ddot{n}}=\sum_{M=0}^{m+\bar{m}} f_{H}^{m \bar{m}_{A_{M}}}\left(z_{p} ; \alpha_{p}\right)
$$

so that

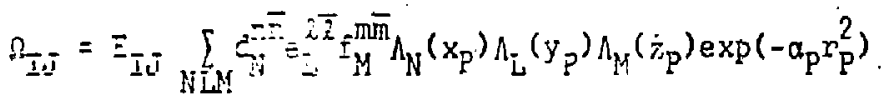

$$
\begin{aligned}
& \text { II is often con:Eatent to write } \Omega_{I J} \text { as. } \\
& \delta_{I J}=\sum_{k} D_{k} n_{N_{k}}\left(x_{P}\right)_{K_{k}}\left(y_{p}\right) n_{M_{k}}\left(z_{p}\right) \exp \left(-\alpha_{p} r_{P}^{2}\right),
\end{aligned}
$$

where

$$
D_{k}=\Sigma_{I J} d_{N_{k}}^{n \bar{n}} e_{L_{k}}^{l \bar{l}} f_{k}^{m \bar{n}} \text {. }
$$

This allows generalization to include spherical harmonic basis functions when $D_{k}$ is replaced by

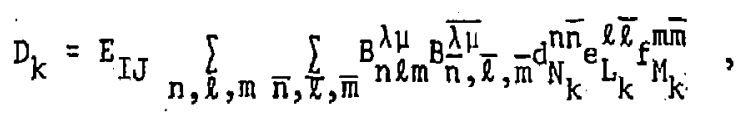

In these equations $k$ indexes all $(N, I, M)$ combinations for which $D$ is non-zero and the charge distribution is specified by a list of the non-zero $D_{k}$ and the corresponding $\left(N_{k}, L_{k}, M_{k}\right)$. In practice the $c^{L}\left(\alpha_{A}\right) c^{\bar{L}}\left(\alpha_{B}\right)$ and normalization are also incorporated into the $D_{k}$. 
Some integrals which must be evaluated involve derivatives of the basis functions. Consequently, it is convenient to define the additional charge distributions

$$
\begin{gathered}
G_{I J}=\phi_{I} \nabla \phi_{J} \\
\cdot T_{I J}=\underline{\nabla}_{I} \cdot \underline{\nabla} \phi_{J}
\end{gathered}
$$

and

$$
Q_{I J}=\left(\underline{\nabla} \phi_{I}\right) \times\left(\underline{\nabla} \phi_{J}\right)
$$

The $x$ component of $G_{I J}$ is easily obtained from

$$
\frac{\partial}{\partial x} \phi\left(\bar{r}, \bar{l}, \bar{n}, a_{B}, B\right)=\bar{n} \phi\left(\bar{n}-1, \bar{l}, \bar{m}, \alpha_{B}, B\right)-2 \alpha_{B} \phi\left(\bar{n}+1, \bar{l}, \bar{m}, \alpha_{B}, B\right)
$$

so that

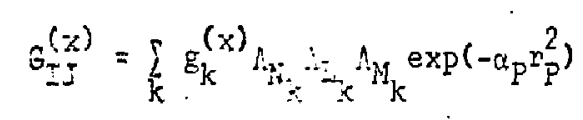

with

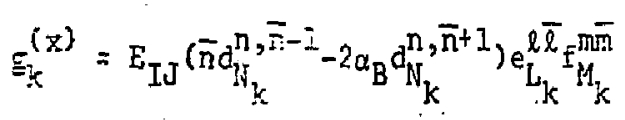

The distribution I IJ may be expanded in an analogous manner to give

with

$$
T_{I J}=\int_{k} t_{k} \Lambda_{N_{k}} \Lambda_{L_{k}} \Lambda_{M_{k}} \exp \left(-\alpha_{p} r_{P}^{2}\right)
$$

$$
t_{k}=\left(t_{k}^{x x}+t_{k}^{y y}+t_{k}^{z z}\right) E_{I J}
$$

$t_{k}^{x x}=\left[\bar{n} \alpha_{N_{k}}^{n-1, \bar{n}-1}-2 n \alpha_{B} d_{N_{k}}^{n-1, \bar{n}+1}-2 \bar{n} \alpha_{A} d_{N_{k}}^{n+1}, \bar{n}-1+4 \alpha_{A} \alpha_{B} d_{N_{k}}^{n+1, \bar{n}+1}\right] e_{L_{k}}^{l \bar{l}} f_{M_{k}}^{n-\bar{m}}$

and similar expressions for $t_{k}^{y y}$ and $t_{k}^{z z}$. The $z$ component of $Q_{I J}$ may be similarly shown to be of the form

$$
Q_{I J}^{(z)}=\sum_{k}^{(z)} \Lambda_{N_{k}} \Lambda_{L_{k}} \Lambda_{M_{k}} \exp \left(-a_{p} r_{p}^{2}\right)
$$


vihere

$$
q_{k}^{(z)}=f_{M_{k}}^{m \bar{m}_{I J}} \mathrm{E}_{k} q_{k}^{x y}
$$

The $q_{k}^{x y}$ obtained directly from $\partial \phi_{I} / \partial x \hat{c} \phi_{J} / \partial y=\partial \phi_{I} / \partial y \partial \phi_{J} / \partial x$ involve new $\phi^{\prime} s$ of total powers of $x, y$, and $z$ higher by two than the starting ones. These highest powers cancel since

$$
\begin{aligned}
& \phi\left(n+1, l, m, \alpha_{A}, A^{\prime}\right) \phi\left(\bar{n}, \vec{l}+1, \bar{m}_{,} \alpha_{B}, B\right)-\phi\left(n, l+1, m, \alpha_{A}, A\right)_{\phi}\left(\bar{n}+1, \vec{l}, \bar{m}_{,} \alpha_{B}, B\right) \\
& =\left(x_{A} y_{B}-y_{A} x_{B}\right) \phi\left(n, l, m, \alpha_{A}, A\right) \phi\left(\bar{n}, \bar{l}, \bar{m}, a_{B}, B\right) \\
& =\left[\overline{A \bar{B}}_{y} \phi\left(n+1, \ell, m, a_{A}, A\right)-\overline{A B}_{x} \phi\left(n, l+1, m, \alpha_{A}, A\right)\right] \phi\left(\bar{n}, \bar{l}, \bar{m}_{B}, a_{B}, B\right)
\end{aligned}
$$

Consequently,

$$
\begin{aligned}
& q_{k}^{x y}=\overline{\bar{l}}\left(\pi d_{N_{k}}^{n-1, \bar{n}_{-}}-2 \alpha_{A} A_{N_{k}}^{n+1}, \bar{n}\right) e_{L_{k}}^{\ell, \bar{l}-1}-2 n \alpha_{B} d_{N_{k}}^{n-1, n_{2}} e_{L_{k}}^{\ell ; \bar{l}+1} \\
& -2\left(\bar{n} d_{N_{k}^{n}, \bar{n}-\bar{z}}-z_{3} d_{N_{k}^{n}, \bar{n}+1}\right) e_{L_{k}}^{l-1, \bar{l}}-2 \bar{n} \alpha_{A} d_{N_{k}}^{n, \bar{n}-1} e_{L_{k}}^{l+1, \bar{l}}
\end{aligned}
$$

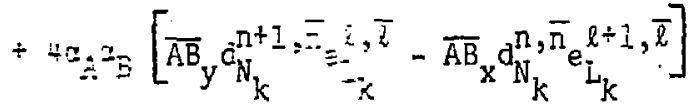

it is important $=s$ note that different sets of $\left(N_{k}, L_{k}, M_{k}\right)$ appear in $2.27,2.28,2.33,2.35$; and 2.38. Also, larger values. of $N+L+M$ appear in the derivatives than in the charge distribution. 
III. BASIC - INTEGRALS

From the previous section it is clear that the one-electron integrals to be evaluated all reduce to the form

$$
\begin{array}{r}
{[N L M \mid \theta]=\int \theta\left(\underline{r}_{1}\right) \Lambda_{N}\left(x_{1 P} ; \alpha_{P}\right) \Lambda_{L}\left(y_{1 P} ; \alpha_{P}\right) \Lambda_{M}\left(z_{1 P} ; \alpha_{P}\right)} \\
\quad \exp \left(-\alpha_{P} r_{1 P}^{2}\right) d \tau_{1}
\end{array}
$$

Likewise the basic two-electron integrals a I take the form

$$
\begin{gathered}
{\left[N L M|\theta| \hat{N L M}^{\prime \prime}\right]=\int \theta\left(\underline{r}_{1}, \underline{r}_{2}\right) \Lambda_{N}\left(x_{1 P} ; \alpha_{P}\right) \Lambda_{L}\left(y_{1 P} ; \alpha_{P}\right) \Lambda_{M}\left(z_{1 P} ; \alpha_{P}\right)} \\
\exp \left(-\alpha_{P} r_{1 P}^{2}\right) \Lambda_{N^{\prime}}\left(x_{2 Q} ; \alpha_{Q}\right) \Lambda_{L},\left(y_{2 Q} ; \alpha_{Q}\right) \Lambda_{M}+\left(z_{2 Q} ; \alpha_{Q}\right) \\
\exp \left(-\alpha_{Q} r_{2 Q}^{2}\right) d \tau_{1} \mathrm{~d} \tau_{2}
\end{gathered}
$$

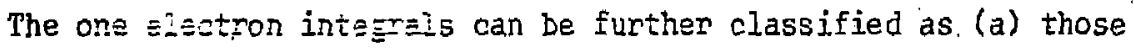
thet can be done in clased form and (b) those that require the same numerically approximated auxiliary functions as the twoelectron integrals.

A. One electron integrals, closed form

The basic integral of this type is the one dimensional integral

$$
\int d x_{\Lambda_{N}}\left(x_{P} ; \alpha\right) \exp \left(-\alpha x_{p}^{2}\right)=\delta_{N, 0}(\pi / \alpha)^{1 / 2}
$$

(the $\delta_{\mathrm{N}, 0}$ arises from the orthogonality of the Hermite polynomials). Thus the overlap integral is simply

$$
[N L M \mid L]=\delta_{N, O} \delta_{L, O} \delta_{M, 0}\left(\pi / \alpha_{p}\right)^{3 / 2}
$$

The relation

$$
x_{C} \Lambda_{N}\left(x_{P} ; a_{P}\right)=N \Lambda_{N-1}+\frac{1}{2} \Lambda_{N+1} / a_{P}+\widetilde{P C}_{X} \Lambda_{N}
$$


then gives

$$
\left[\left.N L M\right|_{C}\right]=\left(\delta_{I V, I}+\overline{P C}_{x} \delta_{N, 0}\right) \delta_{L, 0} \delta_{M, 0}\left(\pi / \alpha_{p}\right)^{3 / 2}
$$

with similar results for $y_{C}$ and $z_{c}$. The second moments are just

$$
\left[N L M \mid x_{\mathrm{C}}^{2}\right]=\left[2 \delta_{\mathrm{N}, 2}+2 \overrightarrow{\mathrm{PC}}_{\mathrm{x}} \delta_{\mathrm{N}, 1}+\left(\overline{\mathrm{PC}}_{\mathrm{x}}^{2}+\frac{1}{2} \alpha_{\mathrm{P}}^{-1}\right) \delta_{\mathrm{N}, 0}\right] \delta_{\mathrm{L}, 0} \delta_{M, 0}\left(\pi ; \alpha_{\mathrm{P}}\right)^{3 / 2}
$$

and

$$
\left[N L M \mid x_{c} y_{c}\right]=\left(\delta_{I, ?} \div \overline{P C}_{x} \delta_{N, 0}\right)\left(\delta_{L, I}+\overline{P C}_{y} \delta_{L, 0}\right) \hat{o}_{M, 0}\left(\pi / a_{P}\right)^{3 / 2}
$$

with simizis results Eor $y_{c}^{2}, z_{c}^{2}, x_{c} z_{c}$ or $y_{c} z_{c}$.

The $\therefore$ inetic $\in n=0 y$ and gradient matrix elemerts are given

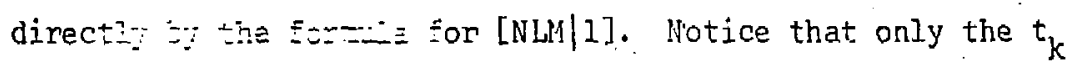

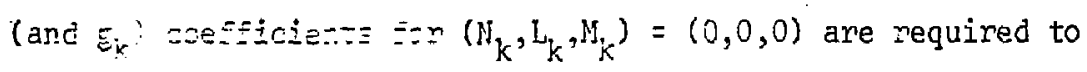

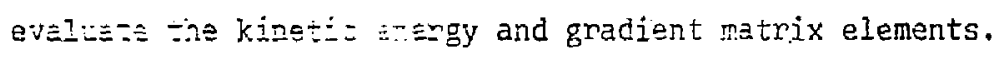

B. Zien one elEz=-2n integruls

FE basic int $\$ \equiv \equiv$ in this category is the nuclear attraction integral $\left[\mathrm{NLM} / \mathrm{r}_{\mathrm{C}}^{-1}\right]$. Enod the definition of the $\Lambda^{\prime} \mathrm{s}$, this may be written $2 \leqq$

$$
\left[\because\left[L M / r_{C}^{-1}\right]=\left(\partial / 3 P_{x}\right)^{N}(\partial / \partial P)^{L}\left(\partial / \partial P_{z}\right)^{M}\left[000 \mid r_{C}^{-1}\right]\right.
$$

The integral $\left[000 / \mathrm{r}_{\mathrm{C}}^{-1}\right.$ ] was shown by Boys to be given by.

$$
\left[000 \mid r_{C}^{-I}\right]=\left(2 \pi / \alpha_{P}\right) F_{o}(T)
$$

where

$$
\mathrm{T}=\alpha_{\mathrm{P}} \overline{\mathrm{CP}}^{2}
$$

and

$$
E_{0}(T)=\int_{0}^{1} \exp \left(-T u^{2}\right) d u
$$


If we define the auxiliary functicn $R_{N L M}^{\prime}$ by

$$
R_{N L M}=\left(\partial / \partial P_{x}\right)^{N}\left(\partial / \partial P_{y}\right)^{L}\left(\partial / \partial P_{z}\right)^{M} F_{0}(T)
$$

then

$$
\left[N L M / r_{C}^{-1}\right]=\left(2 \pi / \alpha_{P}\right) R_{N L M}
$$

The computation of $\mathrm{R}_{\mathrm{NLM}}$ will be described in a later section. Matrix elements of the components of the electric field, such as $x_{c}{ }^{-3} c$ can be evaluated in two ways which illustrate the tricks needed for more complicated integrals. First.

$$
x_{C} C_{C}^{-3}=\partial r_{C}^{-1} / \partial C_{x}
$$

where it should be noted that differentiation is with respect to the melear positin. Therefore

$$
\text { IMIMI } \left.\left.{ }_{2} C_{C}^{-3}\right]=: a C_{x}\right)\left[N L M \mid r_{C}^{-1}\right]
$$

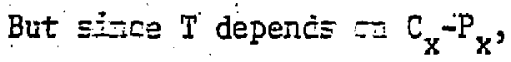

$$
\left(a / \partial C_{x}\right) g(T)=-\left(Z / \partial P_{x}\right) g(T)
$$

for ary function $g(z)$.

Hence,

$$
\left[N L M / X_{C} C^{-3}\right]=-\left(2 \pi / \alpha_{P}\right) R_{N+1, L, M}
$$

Aiternatively this integral can be evluated by noting that

$$
x_{c} c_{c}^{-3}=-\partial x_{C}^{-1} / \partial x
$$

Hence, by integration by parts,

$$
\left[N L M / x_{c} c_{c}^{-3}\right]=\left[\partial(N L M) / \partial \times / x_{C}^{-1}\right]
$$

But, from the definition of $\bar{\Lambda}_{N}$, 


$$
\partial(N, L, M) / \partial x=-(N+I, L, M)
$$

so

$$
\left[N L M \mid x_{C^{2}}{ }^{-3}\right]=-\left(2 \pi / \alpha_{P}\right) P_{N+1,1, M}
$$

The components of the electric field gradient are similarly obtained using

$$
\begin{aligned}
& \left(\partial^{2} / 3 C_{x}^{2}-\partial^{2} / \partial C_{y}^{2}\right) r_{C}^{-1}=3\left(x_{C}^{2}-y_{C}^{2}\right) r_{C}^{-5} \\
& \left(2 a^{2} / \partial c_{z}^{2}-\partial^{2} / \partial c_{x}^{2}-\partial^{2} / \partial c_{y}^{2}\right) r_{C}^{-1}=3\left(3 z_{C}^{2}-r_{C}^{2}\right) r_{C}^{-5}
\end{aligned}
$$

and

$$
\left(z^{2} / \partial C_{x} \partial C_{y}\right) r_{C}^{-1}=3 x_{C} C_{C} r_{C}^{-5}
$$

Notice flese formin are written so as to avoid projisza witn the $= \pm-$ a function which arises in $\nabla^{2} r_{C}^{-1}$. Hence the Ëotric

$$
\begin{aligned}
& {\left[M \mid 3\left(x_{C}^{2}-\cdots=\bar{*}=\left(2 \pi / Q_{P}\right)\left(R_{N+2, L, M^{-R} N, L+2, M}\right)\right.\right.} \\
& {[N L]\left(3=2-j r_{C}^{-5}\right]=\left(2 \pi / \alpha_{P}\right)\left(2 R_{N}, L, M+2-R_{N+2, L, M^{-R}}, I+2, M\right) / 3} \\
& {\left[\text { HIL.M! } \mid 3 x_{C} C_{C}{ }^{-5} C\right]=\left(2 \pi / a_{P}\right) R_{N+I, L}+1, M}
\end{aligned}
$$

Katrix elements over the cre-electron spin-orbit operator may be evaluated by two different methods. If the space part of the basic spin-orbit operator is considered to be $r_{c}^{-3} r_{C} \times \nabla$ then the $z$ comonent, for exampie, is $r_{C}^{-3}\left(x_{C} \partial / \partial y-y_{C} \partial / \partial x\right)$. Matrix elements of this operator can be evaluated by conbining the results given above for the electric field with the $\mathrm{g}_{\mathrm{k}}$ expansion coefficients given previously for the gradient. Alternatively integration by parts gives 


$$
\left\langle\phi_{I}\right| r_{C}^{-3}\left(x_{C} \frac{\partial}{\partial y}-y_{C} \frac{\partial}{\partial x}\left|\phi_{J}\right\rangle=\left[\frac{\partial \phi_{I}}{\partial x} \frac{\partial \phi_{J}}{\partial y}-\frac{\partial \phi_{I}}{\partial y} \frac{\partial \phi_{J}}{\partial x} \mid r_{C}^{-1}\right]\right.
$$

The spin-orbit integral then reduces to using the $q_{k}$ coefficients in summing $\left[\mathrm{NLM} / \mathrm{r}_{\mathrm{C}}^{-1}\right.$ ] matrix elements.

C. Two electron integrals

The simplest integral in this category is the electron repulsion.

$$
\begin{array}{r}
{\left[N L M\left|r_{12}^{-I}\right| N^{\prime} L^{\prime} M^{\prime}\right]=\left(\partial / \partial P_{x}\right)^{N}\left(\partial / \partial P_{y}\right)^{L}\left(\partial / \partial P_{z}\right)^{M}\left(\partial / \partial Q_{x}\right)^{N \prime}\left(\partial / \partial Q_{y}\right)^{L^{\prime}}} \\
\left(\partial / \partial Q_{z}\right)^{M \prime}\left[000 / r_{12}^{-1} \mid 000\right] \quad(3.29)
\end{array}
$$

Boys everuated the sesic integral as

$$
\operatorname{soc} 1 r_{12}^{-1} 1000 i=i_{0}(T)
$$

where

$$
\lambda=2 \pi^{5 / 2} \alpha_{p}^{-1}=-\left(a_{p}+\alpha_{Q}\right)^{-1 / 2}
$$

and

$$
I=\alpha_{P} \alpha_{Q}\left(\alpha_{P}+\alpha_{-}\right)-i \overrightarrow{P Q}^{2}
$$

Becalise $T$ involves only the combination $\underline{P}-\underline{Q}$,

$$
\left(\partial / \partial Q^{x}\right)^{N} g(T)=(-\partial / \partial P)^{N} g(T)
$$

for any Function $g(T)$.

\section{Hence}

$$
\left[N L M\left|x_{22}^{-1}\right| N^{\prime} L^{\prime} M^{\prime}\right]=\lambda(-1)^{N^{\prime}+L^{\prime}+M^{\prime}} R_{N^{\prime}+N^{\prime}, L^{\prime}+L^{\prime}, M^{\prime}+M^{\prime}}
$$

Just as for the electric field and field gradient, integrals over $x_{12} r_{12}^{-3}, x_{12} y_{12} r_{12}^{-5}$, etc., are easily evaluated. For example

$$
\left[N L M\left|x_{12} r_{12}^{-3}\right| N^{\prime} L^{\prime} M^{\prime}\right]=-\lambda(-1)^{N^{\prime}+L^{\prime}+M^{\prime}}{ }_{R^{\prime}+N^{\prime}+1, L+L^{\prime}, M+M^{\prime}}
$$


$\left[N L M\left|3 x_{12} y_{12} r_{12}^{-5}\right| N^{\prime} L^{\prime} M^{\prime}\right]=\lambda(-1)^{N^{\prime}+L^{\prime}+M^{\prime}} R_{N+N^{\prime}+1, L+L^{\prime}+1, M+M^{\prime}}$

$\left[N L M\left|3\left(x_{12}^{2}-y_{12}^{2}\right) r_{12}^{-5}\right| N^{\prime} L^{\prime} M^{\prime}\right]=\lambda(-1)^{N^{\prime}+L^{\prime}+M^{\prime}}\left(R_{N+N^{\prime}+2, L+L^{\prime}, M+M^{\prime}}\right.$

$$
-R_{N+N}, L+L^{\prime}+2, M+M 1^{\prime}
$$

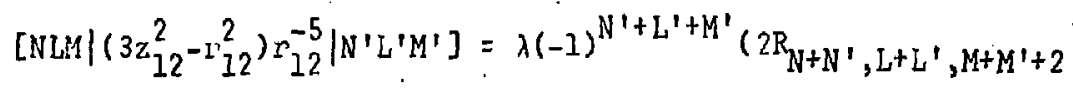

$$
\left.-R_{N+N^{2}+2, L^{+}+L^{\prime}, M+M^{\prime}}-R^{\prime}+N^{\prime}, L+L^{\prime}+2, M^{\prime}+M^{\prime}\right) / 3
$$

Integrels over these $r_{12}^{-5}$ operators appear in calculation of spinspin interaction matrix elements.

The space part : $:$ the two electron spin-orbit operator has the $z==x_{12}^{-3}+12 \times z$. $\quad$ e z component of this operator is then

$$
==\left(x_{12} \frac{\partial}{\partial y_{1}}-\ldots \frac{\partial}{\partial x_{1}}\right) \text {. }
$$

Inte $\approx=$ sion by per:s :-2lds a result similar to that obtained for the ons-ilectron $\because n-\equiv \equiv n \equiv l$,

$$
\begin{aligned}
& \left.\left\langle\dot{\partial}_{I}(I) \phi_{K}(2)\right| F_{I}=x_{I 2} \frac{\partial}{\partial y_{I}}-y_{12} \frac{\partial}{\partial x_{I}}\right)\left|\phi_{J}(1) \phi_{L}(2)\right\rangle \\
& =\left[\frac{\partial \phi_{I}}{\partial x_{I}} \frac{\partial \phi_{I}}{\partial y_{I}}-\frac{\partial \xi_{I}}{\partial y_{I}} \frac{\partial \phi_{I}}{\partial x_{I}}\left|r_{I 2}^{-1}\right| \phi_{K} \phi_{L}\right]
\end{aligned}
$$

Consequently, this integral is given simply as a sum over [NLM $\left.\left|r_{12}^{-1}\right| N^{\prime} L^{\prime} M^{\prime}\right]$ using coefficients $q_{k}$ for the IJ orbitals and $D_{k}$ for the KL onbitals. Alternatively, of course, the integral could be written as a sum over integrals like $\left[N L M\left|x_{12} n_{12}^{-3}\right| N^{1} L^{\prime} M^{1}\right]$ with $g_{k}$ coefficients for the IJ orbitals. 
IV. AUXILIARY FUNCTIONS

\section{A. $\mathrm{R}_{\text {NLM }}$}

The function $R_{N L M}$ is defined as

$$
\cdot R_{N L M}=(\partial / \partial a)^{N}(\partial / \partial b)^{L}(\partial / \partial c)^{M} \int_{0}^{I} e^{-T u^{2}} d u
$$

where

$$
T=a\left(a^{2}+b^{2}+c^{2}\right)
$$

By direct chain-rule differentiation an explicit formula for $R$ can be found:

$$
P_{M I M}=\sum_{n=0}^{[N / 2]} \sum_{2=2}^{[L / 2]} \sum_{m=0}^{[M / 2]} a^{N-2 n_{b} L-2 l c^{M-2 m}}
$$

$$
\frac{M !}{(2 \pi i !(1-2 n) !} \frac{-!}{(2 \lambda i !-2 l) !} \frac{M !}{(2 m) ! !(14-2 m) !} F_{N+L+M-n-l-m}
$$

Fon generatins $\equiv$ sable of all $\mathrm{R}_{\mathrm{NLM}}$ up to some maximum $N+L+M$, as is zeeded in doita slocks of integrals, recursion relations are more useful. T:Ese can be found from introduction of the more gereral integres:

$R_{N L M j}=(-\sqrt{a})^{N+L+M}(-2 \alpha)^{j} \int_{0}^{1} u^{N+L+M+2 j_{H_{N}}(\sqrt{\alpha} a u) H_{L}(\sqrt{\alpha} b u) H_{M}(\sqrt{\alpha} c u) e^{-T u^{2}} d u}$

Let us first note that

$$
\mathrm{R}_{000 j}=(-2 \alpha)^{j} \mathrm{~F}_{j}(T)
$$

where

$$
F_{j}(T)=\int_{0}^{1} u^{2 j} \exp \left(-T u^{2}\right) d u
$$

From the recursion relations for the Hermite polynomials, it follows that

$$
\begin{aligned}
& R_{0,0, M+1, j}=c R_{0,0, M, j+1}+H_{0,0, M-1, j+1} \\
& R_{0, L+1, M, j}=b R_{0, L, M, j+1}+R_{0, L-1, M, j+1}
\end{aligned}
$$




$$
R_{N+1, L, M, j}=\dot{a} R_{N, L, M, j+1}+N R_{N-1, L, M, j+1}
$$

Thus the desired $R_{N L M}$ ' given as $R_{N L M 0}$ ) can be generated from a table of $F_{j}(T)$ for all $j$ between $O$ and the maximum $N+L+M$.

\section{B. $F_{j}$}

Shavitt ${ }^{2}$ has given several cormulas useful for evaluating $F_{j}(T)$. Rapid and accurate evaluation of this function for a wide range of $j$ and $T$ requires some care, however. Our best program at present evaluates $F_{j}(T)$ by different formulas depending on $T$.

For $0<T<12$ and $0 \leq j \leq J, F_{J}(T)$ is first evaluated using the seven tere Taylor exansion

$$
E(Z)=\sum_{x=0}^{0} F_{T-Z}(T)(T * T)^{k} / k !,
$$

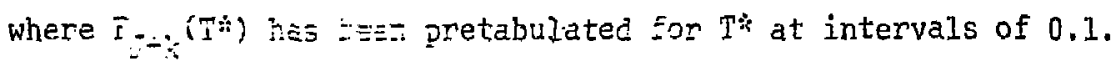
The camonerds $r \in c-r=z=$ relation

$$
E_{i}(Z)=\left[2 T E_{j-1}(Z)+\exp (-T)\right] /(2 j+i)
$$

can then be used to Estain all $F_{j}(T)$.

Fon the renge $22<T<30$ we note that

$$
F_{e}(T)=\frac{1}{2} \sqrt{\pi} / \sqrt{I}-\int_{1}^{\infty} \exp \left(-T u^{2}\right) d u
$$

If the integral $\int_{1}^{\infty} \exp \left(-\mathrm{Tu}^{2}\right)$ du is now expressed as $\exp (-T) g / T$, then $g$ can be computed from

\section{$12<T<15$}

$$
\begin{aligned}
& g=0.4999489092-0.2473631686 \mathrm{~T}^{-1} \\
&+0.321180909 \mathrm{~T}^{-2}-0.3811559346 \mathrm{~T}^{-3}
\end{aligned}
$$


$15<\mathrm{T}<18$

$$
g=0.4998436875-0.24249438 \mathrm{~T}^{-1}+0.24642845 \mathrm{~T}^{-2} \quad(4.13)
$$

$18<\mathrm{T}<24$

$$
g=0.499093162-0.2152832 T^{-1}
$$

$24<\mathrm{T}<30$

$$
g=0.490 \text {. }
$$

Upwards recursion, which is unstable for small $\mathrm{T}$ or large $\mathrm{j}$, can be used to obtain $F_{j}(T)$ with a relative accuracy of $3 \times 10^{-12}$ for $\mathrm{j} \leq 16$ and $\mathrm{T} \geq 12$.

Fon $T>30, F_{0}(T)=\frac{1}{2} \sqrt{\pi} / \sqrt{T}$ combined with upwards recursion is accurate to foureen significant figures. Finally, for $T>2 J+5 i$ the exact $2 a r d s$ recursion

$$
\bar{F} \div-(T)=\left(2 \%--(2 j+I) F_{j}(T)-\exp (-T)\right]
$$

can $\mathrm{D}=$ zeplaced by

$$
E_{j \div I}(I)=(2 T)^{-*}(2 j+1) F_{j}(T)
$$

without Ioss of accurecy. 


\section{v. COMPUTATIONAL CONSIDERATIONS}

The tractability of the two electron integral formulas hinges upon doing all integrals involving foun sets of basis functions concurrently, as all require the same $R \cdot t a b l e$. If the sets are large enough (say all four are p's) then the calculation of $R$ requires only a small fraction of the total time. It then becomes important to perform the loops over basis functions and sums oven contraction terms efficiently.

Table 1 shows ore such scheme. A significant advantage is gained by forming the intermediate array I which allows the actual integral to se Formed outside the sums over $\alpha_{C}$ and $a_{D}$. It is notsionthy $t r \equiv=$ aith only obvious minor modifications this scheme $=\because 5 \leqslant$ empI $=\div \div 0$ calculate spin-spin and spin-orbit two eiszon integn:

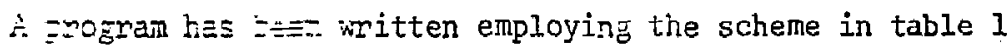
for $t: \equiv$ repulsion $:-:=-j a l s$. Explicit formulas for the elements of $I$ in $=$ ere added for z=-ain $s$ and $p$ integrals. The computation time for a STO-3G set on hydrogen peroxide is $38.5 \mathrm{sec}$ on a. CDC 6400. Dupuis, Rys, and King, ${ }^{7}$ performing the same calculation on a CDC 6400 , obtained times of $8.4 \mathrm{sec}$ for GAUSSIAN $70 ; 39.9 \mathrm{sec}$ for HONDO, a progrem o: their own; and, 132.7 sec for PHANTOM 75, the most recent version of POLYATOM. Upon adding two sets of d functions to the STO-3G set we obtained a computation time of about $124.3 \mathrm{sec}$, somewhat less than HONDO's $152.2 \mathrm{sec}$ and considerably less than PHANTOM's $775.6 \mathrm{sec}$.

It is obvious from these running times that GAUSSIAN 70 is clearly superior for integrals over $s$ and $p$ functions: There are three reasons for this superiority: (1) GAUSSIAN $70^{\prime} \mathrm{s}$ integrals are 
accurate to only 8 figures owing to a less accurate but faster calculation of $I_{m}\left(I^{\prime}\right),(2)$ a coordinate transformation is employed to maximize the local symnetry of the integral over primitive Gaussians, a distinct advantage for highly contracted basis sets; ${ }^{8}$ and ( 3 ) $s$ and $p$ basis functions are combined into one set. GAUSSIAN 70 and HONDO, like our program, compute all integrals over four sets of basis functions (or "shells" in the terminology of Dupuis, Rys and King) concurrently. Judging from the running times this structure has a clear advantage over the one-integralat-a-time method of gHANTOM 75. Our method and the quadrature schere eqployed by تuido seem to be roughly equivalent, at least $=0$ s's and $\Xi^{\prime}$. 
Table 1. Scheme for the computation of all two electron repulsion integrals over $\left[f_{i I A}\right],\left[f_{j J B}\right],\left[f_{k K C}\right]$, and. $\left[f_{l L D}\right] .^{a}$

Sun over $\alpha_{A}$, $\alpha_{B}$

Zero I

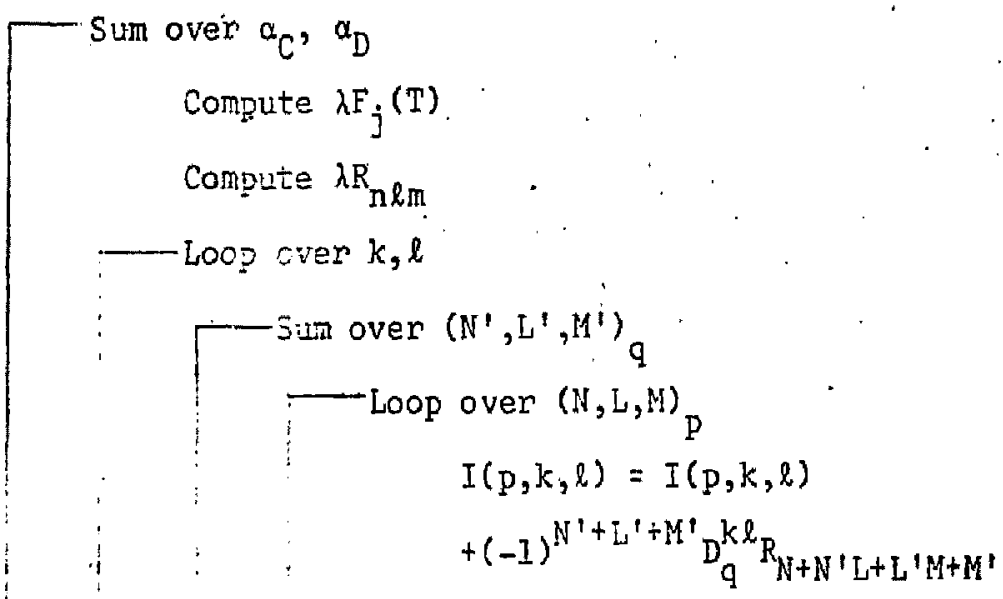

Loop over $k, 2$

$$
\left\{\begin{array}{c}
\text { Loop over } i, j \\
x(i, j, k, l)=x(i, j, k, l)+D_{p}^{i j} I(p, k, l)
\end{array}\right.
$$

${ }^{a} F_{i I A}$ denotes the $i$ th member of set $I$ on center $A$. 
1. S.F. Boys, Proc. Roy. Soc. (London) A200, 542 (1950).

2.. See, for example, Isaiah Shavitt, in Methods in Computational Physics, Vol. 2, edited by B. Alder, S. Fernback, and M. Rotenberg (Academic Press, New York, 1963).pp: 1-45.

3. For a good review, see S. Huzinaga, Supp. Prog. Theo. Phys. 40, 52 (1967).

4. J. L. Whitten, J. Chen, Phys. 39, 349 (1963).

5. S. Huzinaga, J. Chem. Phys. 42, 1293 (1965); T. H. Dunning, Jr. and P.J. Hay, in Modern Theoretical Chemistry, Vol. 3, edited by H. F. Schaefer. III; T. H. Dunning, J. Chen. Phys. 53, 2823 (1970); R. Ditchfield, W. J. Hehre, and J. A. Pople, J. Chem. Phys. 52, 5001 (1970); W. J. Hehwe, R. F. Stewart, and J. A. Pople, J. Chem. Phys. 51, 2657 (1969).

6. Quentun Chemistry Program Exchange, Indiana University, Blocisiston, Inziana.

7. M. I.jpis, J. $=$, and H. F. King, J. Chem. Phys. 65, 111 $(2 \div 5)$.

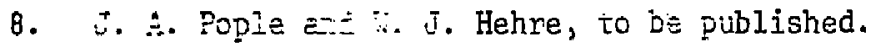

NOTICE

"This repon was prepared as an aceount of work This repon by the United States Govertment. ponsored United States nor the United State Neither the United states nor the their employes Department of Energy, nor any, subcontractors, or not any of their contracton, subcontre express or their employees, makes any wartanty, exp resonimplied, or ansume any legal completeness or sibility fot the accuracy, compretus, product urefulatas of any information, apparatus, produst or process diclosed, or represents that or process disclose ptivalely-owned rights." 